ORIGINAL ARTICLE

Running Author: LIEW ET AL.

\title{
Probing the mechanisms underpinning recovery in post-surgical patients with cervical radiculopathy using Bayesian networks
}

Bernard X. W. Liew ${ }^{1, \square}$

Email liew_xwb@hotmail.com

Anneli Peolsson ${ }^{2}$

Marco Scutari 3

Hakan Löfgren 4,5

Johanna Wibault 2,6,7

Åsa Dedering ${ }^{8,9}$

Birgitta Öberg ${ }^{2}$

Peter Zsigmond ${ }^{10}$

Deborah Falla11

AQ1

AQ2

1 School of Sport, Rehabilitation and Exercise Sciences, University of Essex, Colchester, Essex, United Kingdom

2 Division of Physiotherapy, Department of Medical and Health Sciences, Linköping University, Linköping, Sweden

3 Istituto Dalle Molle di Studi sull'Intelligenza Artificiale (IDSIA), Manno, Switzerland

4 Neuro-Orthopedic Center, Ryhov Hospital, Jönköping, Region Jönköping County, Sweden

5 Department of Clinical and Experimental Medicine, Linköping University, Linköping, Sweden

6 Department of Activity and Health, Linköping University, Linköping, Sweden

7 Department of Medical and Health Sciences, Linköping University, Linköping, Sweden

8 Allied Health Professionals Function, Function Area Occupational Therapy and Physiotherapy, Karolinska University Hospital, Stockholm, Sweden

9 Division of Physiotherapy, Department of Neurobiology, Care Sciences and Society, Karolinska Institutet, Stockholm, Sweden

10 Department of Neurosurgery, Linköping University Hospital, Linköping, Sweden

11 Centre of Precision Rehabilitation for Spinal Pain (CPR Spine), School of Sport, Exercise and Rehabilitation Sciences, College of Life and Environmental Sciences, University of Birmingham, Birmingham, United Kingdom 


\section{Correspondence}

Bernard X. W. Liew, School of Sport, Rehabilitation and Exercise Sciences, University of Essex, Colchester, Essex, United Kingdom.

Emails: bl19622@essex.ac.uk; liew_xwb@hotmail.com

\section{Abstract}

Background Rehabilitation approaches should be based on an understanding of the mechanisms underpinning functional recovery. Yet, the mediators that drive an improvement in post-surgical pain-related disability in individuals with cervical radiculopathy $(C R)$ are unknown. The aim of the present study is to use Bayesian networks (BN) to learn the probabilistic relationships between physical and psychological factors, and pain-related disability in CR.

Methods We analysed a prospective cohort dataset of 201 post-surgical individuals with CR. In all, 15 variables were used to build a BN model: age, sex, neck muscle endurance, neck range of motion, neck proprioception, hand grip strength, self-efficacy, catastrophizing, depression, somatic perception, arm pain intensity, neck pain intensity and disability.

Results A one point increase in a change of self-efficacy at 6 months was associated with a 0.09 point decrease in a change in disability at 12 months $(t=-64.09, p<.001)$. Two pathways led to a change in disability: a direct path leading from a change in self-efficacy at 6 months to disability, and an indirect path which was mediated by neck and arm pain intensity changes at 6 and 12 months.

Conclusions This is the first study to apply BN modelling to understand the mechanisms of recovery in post-surgical individuals with CR. Improvements in pain-related disability was directly and indirectly driven by changes in self-efficacy levels. The present study provides potentially modifiable mediators that could be the target of future intervention trials. BN models could increase the precision of treatment and outcome assessment of individuals with $\mathrm{CR}$.

Trials registration ClinicalTrials.gov identifier: NCT01547611.

Significance Using Bayesian Network modelling, we found that changes in self-efficacy levels at 6-month post-surgery directly and indirectly influenced the change in disability in individuals with CR. A mechanistic understanding of recovery provides potentially modifiable mediators that could be the target of future intervention trials. 

2020

Funding informationThe authors acknowledge financial support from the Swedish Research Council, the Swedish Society of Medicine, the Medical Research Council of Southeast Sweden, Region Östergötland, Lions and Futurum (Academy of Health and Care, Region Jönköping County).

\section{INTRODUCTION}

Cervical radiculopathy $(C R)$ is a prevalent disorder with an incidence of 83 per 100000 individuals, and together with neck pain, ranks fourth in the burden of disease within the United States (Murray et al., 2013; Radhakrishnan, Litchy, O'Fallon, \& Kurland, 1994). The natural history of CR is typically favourable (Radhakrishnan et al., 1994), but individuals who fail to improve may be managed surgically (Bono et al., 2011). While surgery may be effective at resolving pain and neurological symptoms (Bono et al., 2011), its effects on physical recovery in individuals with CR are uncertain (Hermansen, Cleland, Kammerlind, \& Peolsson, 2014; Hermansen, Hedlund, Vavruch, \& Peolsson, 2011; Peolsson, Vavruch, \& Öberg, 2002; Ylinen et al., 2003). Individuals with CR who underwent surgery are known to have persistent deficits in neck muscle endurance, cervical range of motion (ROM) and hand grip strength, relative to agematched healthy controls (Peolsson et al., 2013). These persistent post-surgical physical deficits have been thought to exacerbate pain-related disability in individuals with CR (Engquist et al., 2015; Peolsson et al., 2013; Wibault et al., 2018, 2017). An exercise-based intervention designed specifically to target the aforementioned physical deficits was effective, but not superior to routine clinical care, at improving neck pain-related disability in individuals with CR following surgery (Wibault et al., 2018, 2017).

Psychological features could also be important variables that influence recovery in individuals with CR. For example, in individuals with low back pain, pain catastrophizing (Hall, Kamper, Emsley, \& Maher, 2016), fear avoidance and self-efficacy (Fordham, Ji, Hansen, Lall, \& Lamb, 2017; Mansell, Hill, Main, Vowles, \& Windt, 2016; Spinhoven et al., 2004; Whittle, Mansell, Jellema, \& Windt, 2017) have been reported to be mediators of pain-related disability. Catastrophizing, self-efficacy and psychological flexibility are reported mediators of painrelated disability in individuals with whiplash-associated disorders (WAD) (Andersen, Karstoft, Brink, \& Elklit, 2016; Nieto, Miro, \& Huguet, 2009; Söderlund \& Åsenlöf, 2010; Soderlund, Sandborgh, \& Johansson, 2017; Wicksell, Olsson, \& Hayes, 2010). Yet, no studies have investigated the role of psychological features as mediators of pain-related disability in individuals with CR. In a recent prognostic study on conservatively managed CR, factors that predicted poor recovery of disability after 1 year were the cervical ROM towards the affected side, and the baseline neck disability score (Sleijser-Koehorst et al., 2018). Earlier prognostic studies also reported that cervical ROM, hand grip strength and measures of depression with psychosomatism were important predictors of long-term disability, albeit in post-surgical patients with CR (Peolsson \& Peolsson, 2008; Peolsson, Vavruch, \& Öberg, 2006).

An understanding of the mediators of recovery of pain-related disability would enable researchers and clinicians to better design specific interventions to manage a complex disorder such as CR. The first aim of the present study was to model the probabilistic relationships between physical and psychological factors, and pain-related disability in individuals with CR, post-surgery. For the second aim, we wanted to use the model to understand the mediators (if any) of pain-related disability. We used Bayesian networks (BN) to "learn" and quantify the relationships between multiple variables (Scutari, Auconi, Caldarelli, \& Franchi, 2017), and the 
ensuing model can be used for a causal analysis of the mechanisms underpinning the recovery of disability in individuals with CR. Based on the best available literature in CR and other neck pain disorders, we hypothesized that psychological features, such as catastrophizing, selfefficacy, depression and psychosomatic scores, as well as features of physical function, such as cervical ROM and hand grip strength, would mediate the relationship between pain and disability.

\section{METHODS}

\subsection{Study design}

The present analysis was undertaken on a prospective cohort dataset collected from a multicentre, parallel-grouped, randomized controlled trial, the methodological details of which have been previously reported (Peolsson et al., 2014; Wibault et al., 2018, 2017). This study received approval from the regional ethics review board in Linköping (Dnr M126-08) and was conducted in accordance with the Declaration of Helsinki.

\subsection{Participants}

Eligible patients with CR referred for surgery at four spinal centres in the south of Sweden were selected for the present study, if they fulfilled all of the following inclusion criteria: aged 1870 years old, persistent CR symptoms $\geq 2$ months (median [25th to 75 th percentile] arm pain duration of 12 [9-24] months), unsatisfactory improvement after non-surgical treatments, and magnetic resonance imaging results of disc disease that was compatible with clinical findings. Patients were excluded if they had previous neck surgery, cervical column fracture or traumatic subluxation, cervical myelopathy, malignancy or spinal tumours, spinal infection, any disorders which contraindicate the performance of an extensive rehabilitation programme, myofascial pain syndromes, persistent or recurrent severe back pain, diagnosis of a severe psychiatric disorder, such as schizophrenia or psychosis, drug or alcohol addiction and lack of fluency in Swedish (Peolsson et al., 2014).

\subsection{Surgery}

The present analysis pooled data from both intervention groups, to form a single prospective cohort. Hence, the variable of "intervention group" was not included in the present analysis. The variable "intervention group" was excluded since both groups had an equivalent outcome for their neck-pain-related disability (Wibault et al., 2018). The type of surgery and the postoperative clinical care received are both summarized for descriptive purposes.

A total of 201 participants (mean [standard deviation $(S D)$ ] age $=50.0$ [8.4] years, males $=105$, females $=96$ ) were included. In all, 163 participants received an anterior cervical discectomy and fusion (ACDF), whereas 38 participants received a posterior cervical foraminotomy with or without laminectomy. The type of surgery received by each participant was individually determined by the surgeons at each of the four spinal centres, based on the patient's clinical presentation (Wibault et al., 2017).

\subsection{Interventions}

Differences in the clinical outcomes of the two post-operative rehabilitation approaches have been previously reported (Wibault et al., 2018, 2017).

\subsubsection{Common post-surgical care (weeks 1-6 post-surgery)}


For the first 6 weeks immediately post-surgery, all participants followed an identical rehabilitation pathway (Wibault et al., 2018, 2017). The pathway included advice about appropriate ergonomics and posture, movement tasks to avoid during the first post-surgical week, and instructions about shoulder mobility exercises. No neck collar was prescribed. On the 6th week, patients returned for a final routine visit to the spinal centre with the surgeon and a physiotherapist; the latter instructing patients about the performance of neck mobility exercises. After the 6th week post-operative visit, participants were randomly allocated to either a structured post-surgical rehabilitation or a standard post-surgical rehabilitation group (Wibault et al., 2018, 2017).

\subsubsection{Structured post-surgical rehabilitation (weeks 7-26)}

Participants in this group were referred to a primary care physiotherapist local to each participant's residential setting. Each physiotherapist received a half day practical training session with the project leader with written and oral communications on the rehabilitation programme. The structured physiotherapy programme was based on the management of other neck pain disorders, and included both a neuromuscular training component and a cognitivebehavioural component (Gross et al., 2015; Monticone et al., 2015). The neuromuscular training component started with retraining the activation of the deep neck muscles, which progressed into isometric and dynamic exercises focused on improving the pattern and endurance of neck muscle activation. Participants also performed exercises designed to improve the control and endurance of the shoulder and trunk muscles. The cognitive-behavioural component focused broadly on goal setting, pain neurophysiology education, coping strategies (e.g. strategies such as relaxation training, breathing techniques when symptoms worsen) and stress management (Peolsson et al., 2014). Between weeks 6 and 12, patients visited the physiotherapists once per week. Between weeks 13 and 26, the number of physiotherapy visits increased to twice per week. Participants were also advised on the performance of a home exercise programme. At week 27, participants were discharged and were encouraged to continue with their home exercise programme and to continue increasing their physical activity levels.

\subsubsection{Standard post-surgical rehabilitation (weeks 7-26)}

Participants in this group were treated in accordance with the Swedish usual post-surgical care of individuals with CR. Briefly, patients were referred to their local physiotherapist on an asneeded basis, decided by the patients themselves. Any physiotherapy interventions were pragmatic and not designed specific to rehabilitate known neuromuscular deficits of neck pain disorders.

\subsection{Data collection}

All continuous variables (i.e. variables 3-13 below) were assessed at baseline (pre-surgery), 6and 12-month follow-up. The maximum proportion of missing data was at $37.8 \%$ for the neck proprioception data at 12-month follow-up. The number of participants with complete missing data of variables 3-13 at baseline, 6 months and 12-month follow-up were 0, 14 and 27, respectively. Reasons for the missing data can be found in previous reports of the study (Wibault et al., 2018, 2017).

\subsection{Outcome measures}

The following 13 variables were used to form a BN:

1. Sex: men or women.

2. Age: in years. 
3. Total neck endurance (NeckEndr): cervical extensor and flexor timed endurance were measured in the prone and supine position, respectively (Peolsson et al., 2013). Total endurance (seconds) was calculated by adding extensor and flexor endurance.

4. Total hand strength (HandStr): a Jamar hand dynamometer was used to measure isometric grip strength bilaterally (Peolsson et al., 2013). Total hand strength (kg) was calculated by combining left- and right-hand strength.

5. Total range of motion (TotROM): active cervical ROM in all three cardinal planes were measured with a cervical ROM device in a seated position (Peolsson et al., 2013). The total ROM $\left({ }^{\circ}\right)$ was calculated by adding ROM from all six directions.

6. Average neck proprioception (Propriop): a measure of the ability to return the head to a neutral head posture from $30^{\circ}$ of cervical rotation with the eyes closed. Neck proprioception was tested across three repetitions, twice following both right and left cervical rotation. Proprioception $\left({ }^{\circ}\right)$ was averaged across the three repetitions.

7. Self-efficacy scale (SES): a measure to evaluate each participant's confidence in their ability to perform 20 activities of daily living. Score ranges from 0 (not confident) to 200 (very confident) (Altmaier, Russell, Kao, Lehmann, \& Weinstein, 1993; Bunketorp, Carlsson, Kowalski, \& Stener-Victorin, 2005).

8. Coping strategy questionnaire, catastrophizing subscale (CSQ-CAT): the catastrophizing subscale (questions $5,12,14,28,38$ and 42) of the Swedish version of the CSQ (Jensen \& Linton, 1993) was used to assess negative thinking. Score ranges from 0 (no catastrophizing) to 36 (maximal catastrophizing).

9. Modified Zung self-rating depression scale: measures the level of depressive symptoms. Score ranges from 0 (no depression) to 69 (severe depression) (Zung, 1965).

10. Modified somatic perception questionnaire (MSPQ): measures the magnitude of heightened somatic awareness and anxiety (Main, 1983). Score ranges from 0 (no heightened somatic awareness) to 39 (maximal heightened awareness).

11. Neck pain intensity: a self-reported measure of current neck pain intensity on the visual analogue scale (VAS). Score ranges from 0 (no pain) to 100 (worst imaginable pain).

12. Arm pain intensity: a self-reported measure of current arm pain intensity on the VAS. Score ranges from 0 (no pain) to 100 (worst imaginable pain).

13. Neck disability index (NDI): a measure to quantify disability attributed to neck pain. Score ranges from 0 (no activity limitations) to 50 (maximal activity limitations).

The physical variables (variables 3-7) were presently included as previous studies have reported persistent deficits in these variables in individuals with CR post-surgery, compared to healthy controls and are thought to be associated with persistent disability in the former cohort (Engquist et al., 2015; Peolsson et al., 2013; Wibault et al., 2018, 2017). Psychological variables (7-10) were presently included as these variables have been reported to be either mediators or prognostic factors of disability recovery in musculoskeletal pain disorders (Fordham et al., 2017; Hall et al., 2016; Marshall, Schabrun, \& Knox, 2017; Peolsson et al., 2006).

\subsection{Approach to data analysis}

Descriptive summary measures of mean and $S D$ for all continuous variables 3-13 as described above were calculated for each follow-up time point. All analyses codes and data are included in the Supplementary Material.

\subsubsection{Unfolding of repeated outcome measures}

We modelled the change scores of outcomes 3-12 between 6 months and baseline (i.e. difference between 6 month scores from baseline), and between 12 and 6 months (i.e. 
difference between 12 month scores from 6 month) of the physical and psychosocial variables, to understand the response to treatment over time. The change scores between 6 months and baseline are suffixed with "d6," while the change scores between 12 and 6 months are suffixed with "d12." For the variable of NDI, we used the change scores between baseline and 12 months (suffixed with "final"). The absolute values of age at baseline, and sex was used in the BN analysis (suffixed with "base"). The specific nature of the unfolding enabled us to quantify which variables needed to change and when, to alter the reduction in pain-related disability.

\subsubsection{Bayesian network analysis}

All analyses were performed in R software using the bnlearn package (Scutari, 2010). BN is a graphical modelling technique (Nagarajan, Scutari, \& Lèbre, 2013), used increasingly in the health sciences to understand causal relationships (Farmer, 2014; Sesen, Nicholson, BanaresAlcantara, Kadir, \& Brady, 2013; Takenaka \& Aono, 2017; Zhang et al., 2015). BN are able to handle missing data (Friedman, 1997), which makes them practical in settings where patient records are often incomplete. BN quantifies the relationships among a set of variables $X=\left\{X_{1}\right.$, $\left.\ldots, X_{N}\right\}^{*}$, where $N$ is the number of different variables, using a directed acyclic graph (DAG). Each variable is associated with a node and directed arcs represent conditional dependencies between pairs of nodes. Building a BN model using a data-driven approach involve two stages: (a) structural learning-identifying which arcs are present in the DAG and (b) parameter learning-estimating the parameters that regulate the strength and the direction of the corresponding relationships.

BN can easily include prior knowledge, sourced from the literature and experts, during the model building process. In the BN framework, prior knowledge can be included in the model as blacklist and whitelist arcs. Blacklist arcs are those which contravene known biological/physical mechanisms. For example, depression does not influence age. We blacklisted all arcs which point backwards in time (e.g. from ArmPain_d12 to ArmPain_d6). We also blacklisted arcs pointing between the nodes of age and sex; and pointing from NDI to all other variables-since we were interested in understanding the mediators of pain-related disability as an outcome. Whitelisted arcs are those where there is knowledge from the literature, experts, or where the mechanisms for supporting such arcs are realistic. We included arcs as whitelist connecting the change scores for each variable (variables 3-12) from 6 months (d6) to the change scores of the same variable at 12 months (d12). Such whitelisted arcs represent a form of temporal correlation structure, such as in an auto-regressive process.

We made use of model averaging to reduce the number of arcs that are incorrectly included in the BN. We used the most common implementation of model averaging, that consists of resampling the data multiple times $(B=200)$ using bootstrap and performing structure learning on each of the resulting sample using structural expectation-maximization (Friedman, 1997). We computed an "average" consensus DAG by selecting those arcs that have a frequency of $>70 \%$ in the bootstrapped samples, to create a sparse and interpretable network (Scutari \& Nagarajan, 2013). We randomly split the data $(n=201)$ into a training set $(n=181,90 \%)$ and a testing set $(n=20,10 \%)$, and performed structural and parameter learning on the training dataset. We used the BN model learned from the training set to perform validation on the testing set by computing the correlation coefficient between the predicted and observed values of each continuous variable. The strength of correlation was categorized as negligible $(|r| \leq .30)$, low $(|r|=.31-.50)$, moderate $(|r|=.51-.70)$, high $(|r|=.71-.90)$ and very high $(|r|=.91-1)$ (Hinkle, Wiersma, \& Jurs, 2003). A model with high predictive performance should have as high a positive correlation as possible in the testing dataset. 


\subsubsection{Conditional probability queries}

The derived averaged BN model can be considered an "expert system," which means that we can elicit a sample of realizations of the modelled variables under specific conditions. For example, we can query the system to infer the values of NDI_final when NeckPain_d6 reduced by a threshold value. For each conditional probability query, we sampled $10^{4}$ realizations of the variables of interest to obtain precise probability estimates. We used a technique known as belief updating, which estimates the posterior probability of an event happening based on the available evidence on the values of certain variables. In particular, we adopted a specific method of belief updating known as logic sampling (Nagarajan et al., 2013).

\section{RESULTS}

The mean (SD) values for all continuous variables 3-13 at each follow-up time point is reported in Figure 1. Bivariate relationships between the change scores for variables 3-13 can be found in Figure 2. Figure 3 shows the averaged BN consensus model learnt from 200 networks constructed from the data, with arcs appearing at least in $70 \%$ of the networks kept. The predictive correlations for all variables are included in Table 1.
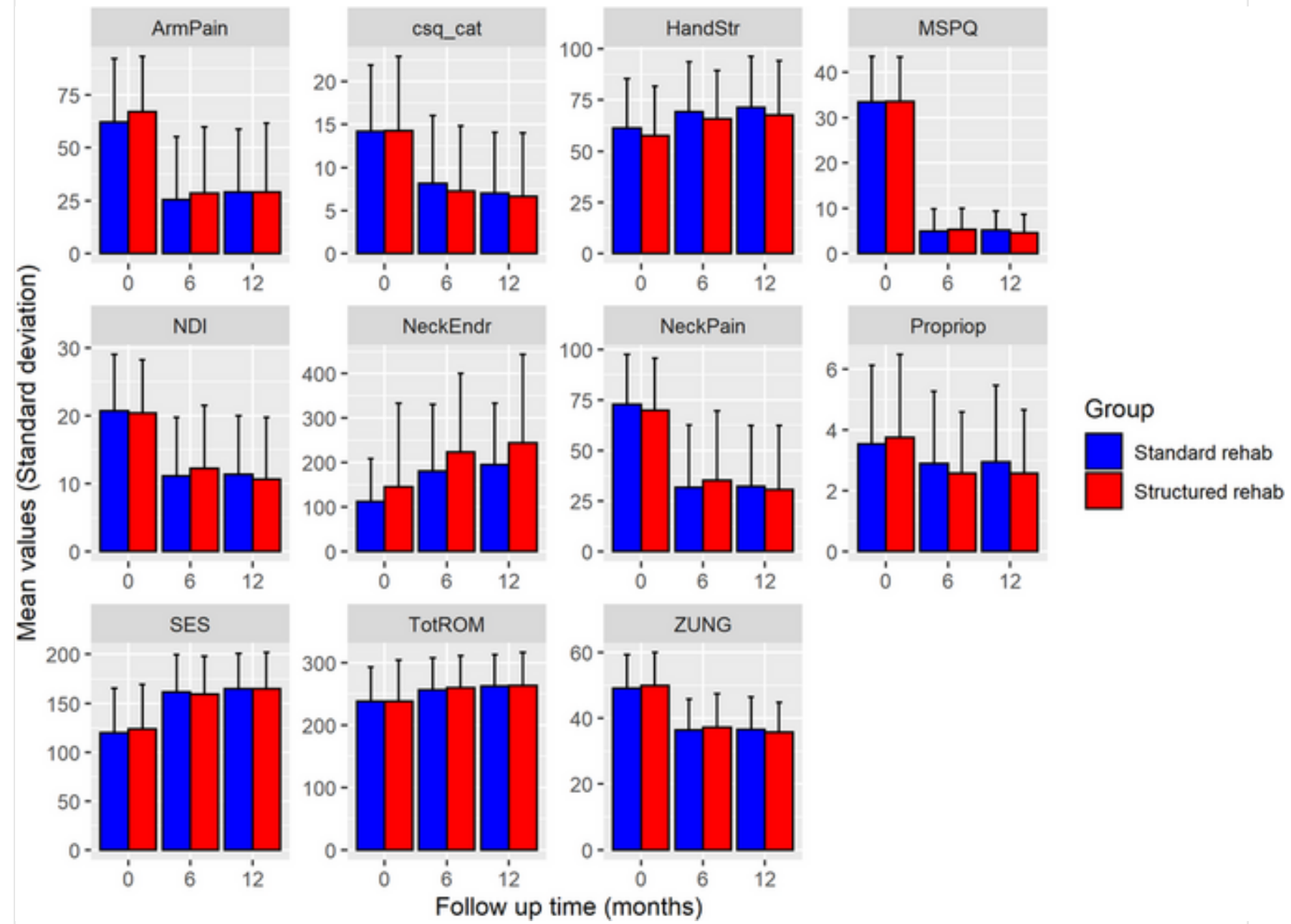

Fig. 1 Mean and standard deviation of clinical variables used in Bayesian Network model. ArmPain, arm pain intensity; csq_cat, coping strategy questionnaire, catastrophizing subscale; HandStr, total hand strength; MSPQ, modified somatic perception questionnaire; NDI, neck disability index; NeckEndr, total neck muscle 
endurance; NeckPain, neck pain intensity; Propr, averaged neck proprioception; SES, Self-Efficacy Scale; TotROM, total range of motion; ZUNG, self-rating depression scale

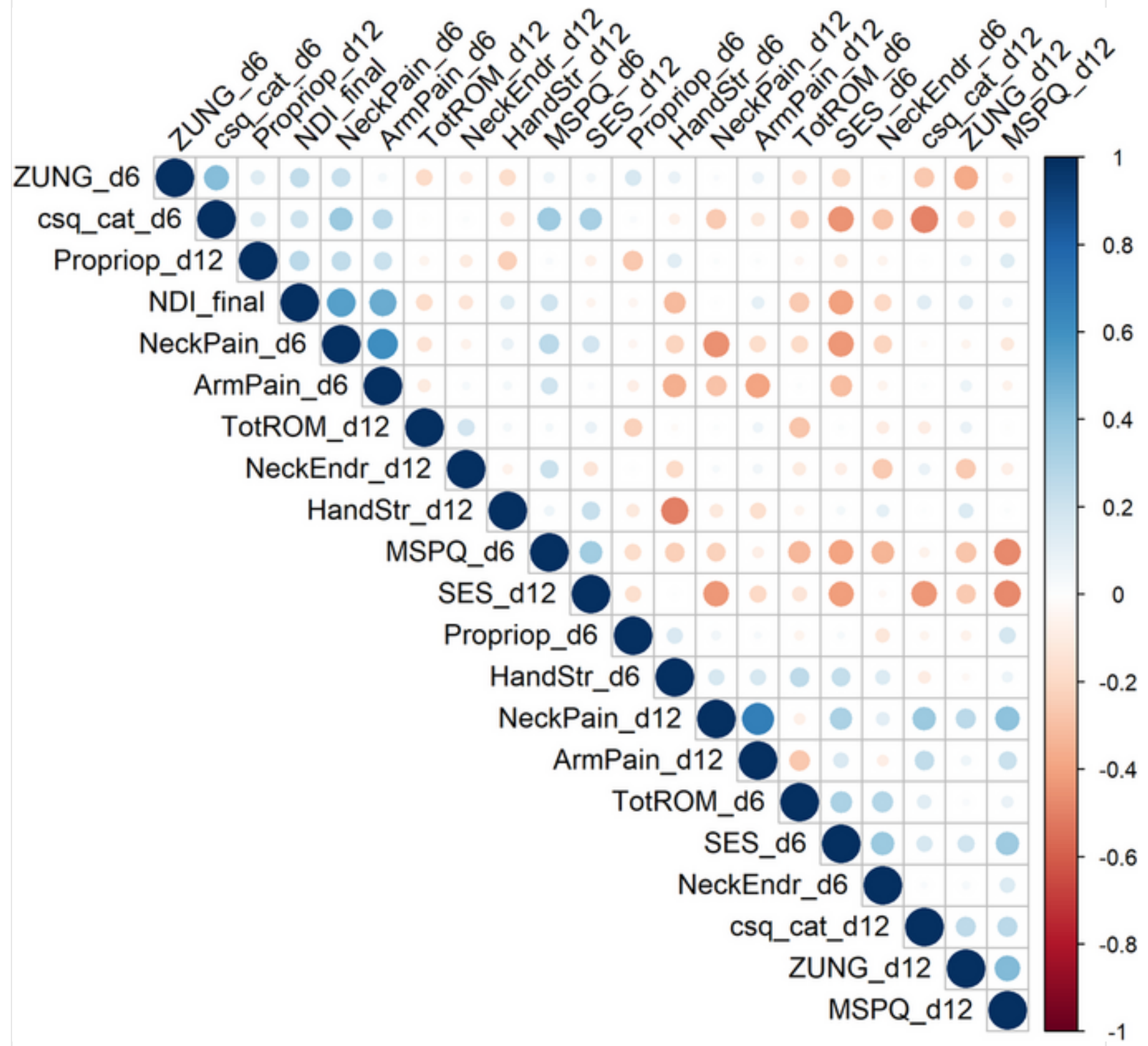

Fig. 2 Pairwise bivariate relationships (Pearson's correlation) between clinical variables used in the Bayesian Network model. Correlation was calculated on instances with complete data. _d6, change from baseline to 6-month follow-up; _d12, change from baseline to 12-month follow-up; _final, change scores between baseline and 12 months; ArmPain, arm pain intensity; csq_cat, coping strategy questionnaire, catastrophizing subscale; HandStr, total hand strength; MSPQ, modified somatic perception questionnaire; NDI, neck disability index; NeckEndr, total neck muscle endurance; NeckPain, neck pain intensity; Propr, averaged neck proprioception; SES, Self-Efficacy Scale; TotROM, total range of motion; ZUNG, self-rating depression scale 


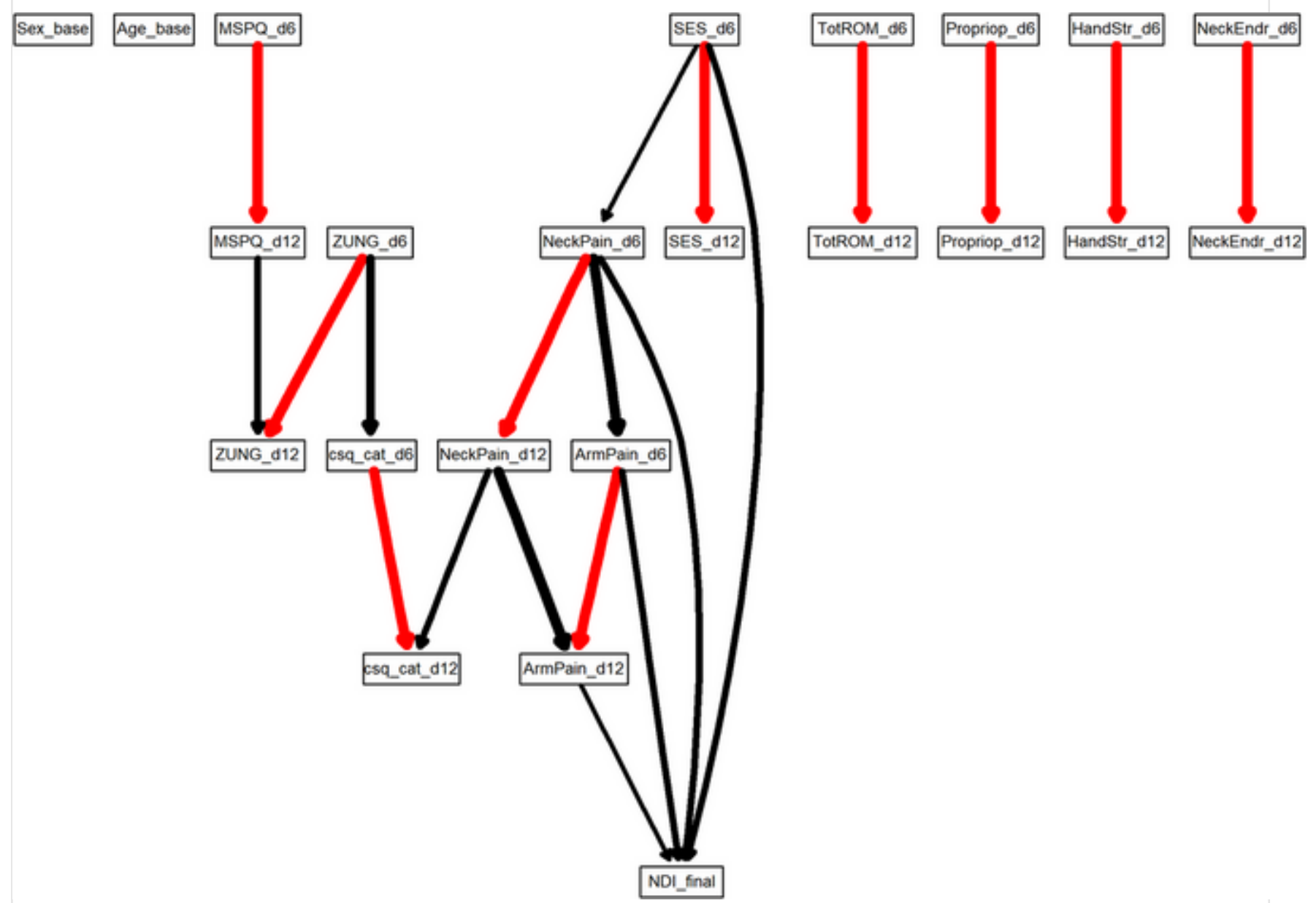

Fig. 3 The directed acyclic graph (DAG) underlying the consensus Bayesian Network of learned from the variables across 201 participants. The thickness of the arcs is in proportion to their strength. Arcs in red are enforced to be present in the network by the whitelist. Only arcs with strength $>0.7$ are included in the consensus network. _d6, change from baseline to 6-month follow-up; _d12, change from baseline to 12-month follow-up; _final, change scores between baseline and 12 months; ArmPain, arm pain intensity; csq_cat, coping strategy questionnaire, catastrophizing subscale; HandStr, total hand strength; MSPQ, modified somatic perception questionnaire; NDI, neck disability index; NeckEndr, total neck muscle endurance; NeckPain, neck pain intensity; Propr, averaged neck proprioception; SES, Self-Efficacy Scale; TotROM, total range of motion; ZUNG, self-rating depression scale

Table 1 Correlation values between observed and predicted variables in the testing subset of data

\begin{tabular}{|l|l|l|}
\hline Variables & Correlation & Strength \\
\hline ZUNG_d6 & .31 & Low \\
\hline MSPQ_d6 & .8 & High \\
\hline SES_d6 & .83 & High \\
\hline
\end{tabular}




\begin{tabular}{|l|l|l|}
\hline Variables & Correlation & Strength \\
\hline csq_cat_d6 & .69 & Moderate \\
\hline TotROM_d6 & .21 & Negligible \\
\hline Propriop_d6 & .58 & Moderate \\
\hline HandStr_d6 & -.05 & Negligible \\
\hline NeckEndr_d6 & .59 & Moderate \\
\hline ArmPain_d6 & .79 & High \\
\hline NeckPain_d6 & .85 & High \\
\hline ZUNG_d12 & .54 & Moderate \\
\hline MSPQ_d12 & .75 & High \\
\hline SES_d12 & .58 & Moderate \\
\hline csq_cat_d12 & .99 & Very high \\
\hline TotROM_d12 & .26 & Negligible \\
\hline Propriop_d12 & .59 & Moderate \\
\hline HandStr_d12 & -.14 & Negligible \\
\hline NeckEndr_d12 & .57 & Moderate \\
\hline ArmPain_d12 & .74 & High \\
\hline NeckPain_d12 & .87 & \\
\hline NDI_final & & \\
\hline
\end{tabular}

Abbreviations:_d12, change from baseline to 12-month follow-up; _d6, change from baseline to 6-month follow-up; final, change scores between baseline and 12 months; ArmPain, arm pain intensity; csq_cat, coping strategy questionnaire, catastrophizing subscale; HandStr, total hand strength; MSPQ, modified somatic perception questionnaire; NDI, neck disability index; NeckEndr, total neck muscle endurance; NeckPain, neck pain intensity; Propr, averaged neck proprioception; SES, Self-Efficacy Scale; TotROM, total range of motion; ZUNG, self-rating depression scale.

\subsection{Pathway(s) leading to a reduction in pain-related disability}

Two pathways led to a change in NDI_final: a direct path from SES_d6 to NDI_final, and an indirect path from SES_d6, passing through NeckPain ( $d 6$ and d12) and ArmPain (d6 and d12) (Figure 3, 4a). From the sampled posterior distribution, a one point increase in SES_d6 was 
associated with a 0.10 point decrease in NDI_final $(t=-64.09, p<.001)$. The probability of having a greater than 50th percentile reduction in NDI_final increased from $4 \%$ if SES_d6 worsened (i.e. $<0$ points), to $47 \%$ if SES_d6 improved (i.e. $>0$ points). A one point increase in SES_d6 was associated with a 0.37 percentage point reduction in NeckPain_d6 ( $t=-46.61$, $p<.001)$. A one percentage point reduction in NeckPain_d6 was associated with a 0.12 reduction in NDI_final $(t=68.89, p<.001)$ and with a 0.56 percentage point reduction in ArmPain_d6 $(t=74.42, p<.001)$.

\subsection{Simulating an intervention for mediation analysis}

We simulated a scenario where neck pain was not dependent on self-efficacy change at 6 months, by fixing the value of the NeckPain_d6 regression coefficients in the local distributions to zero, which removed the SES_d6-NeckPain_d6 arc. When fixing the value of NeckPain_d6 to zero (i.e. the only path from SES_d6 to NDI_final is the direct path), a one point increase in SES_d6 was associated with a 0.07 point decrease in NDI_final $(t=-47.99, p<.001)$ (Figure $4 b$ ). When fixing the values of ArmPain_d12 to zero (i.e. only the direct path from NeckPain_d6 to NDI_final remained), a one percentage point reduction in NeckPain_d6 was associated with a 0.09 reduction in NDI_final $(t=53.38, p<.001)$; and a one point increase in SES_d6 was associated with a 0.09 point decrease in NDI_final ( $t=-60.94, p<.001$ ) (Figure 4c).

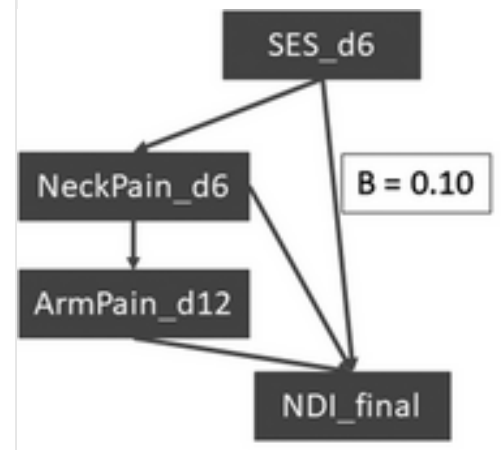

a Total SES_d6 effect

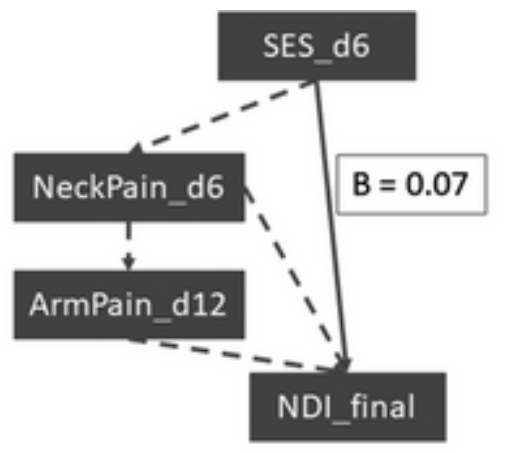

b $70 \%$ of SES_d6 effect

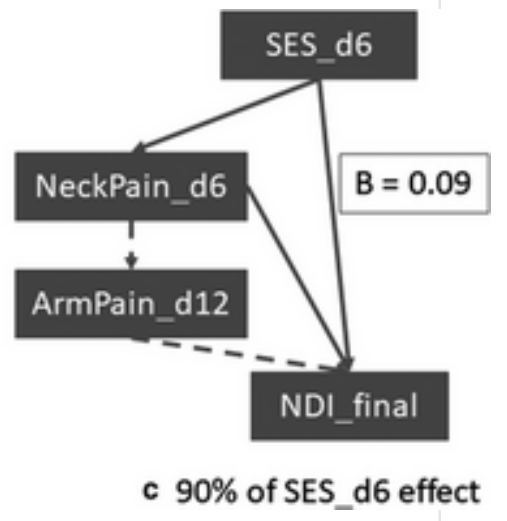

c $90 \%$ of SES_d6 effect

Fig. 4 Contribution of direct and indirect pathways from SES_d6 to NDI_final. (a) Total effect SES_d6 has on NDI_final, (b) direct effect SES_d6 has on NDI_final and (c) effect SES_d6 has on NDI_final after fixing ArmPain_d12. _d6, change from baseline to 6month follow-up; _d12, change from baseline to 12-month follow-up; _final, change scores between baseline and 12 months; ArmPain, arm pain intensity; NDI: Neck disability index; NeckPain, neck pain intensity; SES, Self-Efficacy Scale. Dotted lines = removed arc through simulation.

The $\beta_{\text {SES_d } 6}$ for the outcome of NDI_final reduced from 0.10 to 0.07 , after removing the indirect pathway passing through NeckPain_d6; and reduced from 0.10 to 0.09 after removing the indirect pathway passing through ArmPain_d12. Hence, 30\% of the influence of self-efficacy change on disability was due to the simultaneous reduction of neck pain and/or arm pain, $10 \%$ was due to an eventual reduction in arm pain and $20 \%$ was due to the reduction in neck pain alone (Figure 4). The $\beta_{\text {NeckPain_d6 }}$ for the outcome of NDI_final reduced from 0.12 to 0.09 , after removing the indirect pathway passing through ArmPain_d12. 25\% of the influence of neck pain reduction at 6 months on disability was due to the reduction of arm pain.

\section{DISCUSSION}


Compared to several other musculoskeletal disorders, the mediators that drive an improvement in pain-related disability in individuals with $C R$ is unknown. We used BN to learn from data, the probabilistic relationships between physical and psychological factors, and pain-related disability. Contrary to our hypothesis, physical and psychological factors did not mediate the pain-disability relationship. However, neck and arm pain partially mediated the relationship between improvements in self-efficacy and pain-related disability.

Surprisingly, self-efficacy was the only psychological factor which influenced pain-related disability. A lack of mediation analysis studies in the area of CR meant that we have to compare our findings with that of other painful disorders. In a heterogeneous group of pain disorders, self-efficacy has been shown to either mediate the relationship from pain intensity to depressive symptoms (Arnstein, Caudill, Mandle, Norris, \& Beasley, 1999; Cheng et al., 2018; Craig et al., 2013), or from depressive symptoms to pain intensity (Skidmore et al., 2015). This was in contrast to the present finding that changes in self-efficacy levels were not related to changes in depressive symptoms, but the former was critical in its influence on both neck pain intensity and disability. At the associative level, many studies which investigated post-surgical recovery in individuals with $\mathrm{CR}$ have reported depressive symptoms to be an important predictor of disability (Li, Qi, Yuan, \& Chen, 2015; Peolsson et al., 2006; Persson \& Lilja, 2001; Skeppholm, Fransson, Hammar, \& Olerud, 2017). A limitation of these studies was that depressive symptoms was not investigated together with other psychological factors (Li et al., 2015; Peolsson et al., 2006; Persson \& Lilja, 2001; Skeppholm et al., 2017). This means that the predictive influence of depressive symptoms on disability, after accounting for the simultaneous influence of other psychological factors, remains uncertain.

Reduction in levels of neck and arm pain has been thought to be important factors influencing the recovery of disability in individuals with CR (Engquist et al., 2015; Passias et al., 2018). However, the reduction in neck and arm pain explained only a third of the influence of selfefficacy on the recovery of disability. This means that the mechanism of the direct pathway between self-efficacy and disability remains to be established. In a study on individuals with WAD, two factors were hypothesized to mediate the relationship between self-efficacy and disability: ability to decrease pain and coping with pain (Soderlund et al., 2017). Both mediators were found not to have mediated the strong direct effect self-efficacy had on disability (Soderlund et al., 2017). The direction relationship between self-efficacy and disability has been consistently reported in spinal pain disorders (Lee et al., 2015; Mansell, Kamper, \& Kent, 2013), suggesting that it is a clinically important variable to consider in the clinical management of CR. It may be that individuals with higher self-efficacy engaged in adaptive behaviours to a greater extent, such as exercises, which improved disability more than individuals with lower selfefficacy.

An interesting question that remains to be explored is what facets of disability are differently influenced by direct changes in self-efficacy, indirectly by factors such as neck and arm pain, or by latent factors not included in the present study's model. This is because outcome measures such as the NDI, evaluates the response to 10 different domains (Steinhaus et al., 2019). A clearer understanding of the causal mechanisms of recovery of physical function in people with painful disorders may first require a more precise definition and operationalization of the construct. Towards this end, directly quantifying physical function can be achieved using wearable accelerometers to quantify physical activity levels in naturalistic settings (Pedler, Kamper, Maujean, \& Sterling, 2018). 
The smaller contribution of arm pain recovery, compared to neck pain recovery, on the recovery of disability had indirect support from the literature. A previous study reported that the minimum clinically important difference (MCID) after ACDF was $\frac{4.1}{10}$ points for arm pain, and $\frac{2.6}{10}$ for neck pain (Parker, Godil, Shau, Mendenhall, \& McGirt, 2013). This suggests that a unit recovery of neck pain is clinically more important than a unit recovery in arm pain in individuals with CR. The odds ratio (OR) of predicting patient satisfaction after surgery in individuals with $C R$ was greater when the recovery of neck pain exceeded the MCID $(O R=3.42)$ than when the recovery of arm pain exceeded the MCID (OR = 2.01) (Andresen et al., 2018). The present cohort had on average similar levels of baseline neck and arm pain (VASneck $=\frac{71.4}{100}$, VASarm $=\frac{64.8}{100}$ ). The relative importance of neck versus arm pain recovery on disability may differ between clinical sub-groups where neck pain may exceed arm pain, and vice versa.

Physical factors such as ROM, neck muscle endurance, neck proprioceptive ability and hand grip strength, did not influence the change in disability, which is in disagreement with previous studies (Halvorsen, Kierkegaard, Harms-Ringdahl, Peolsson, \& Dedering, 2015; Peolsson \& Peolsson, 2008; Wibault et al., 2014). Baseline cervical ROM and self-efficacy values were predictors of baseline disability in individuals with CR (Wibault et al., 2014). However, the predictive capacity of self-efficacy $(\beta=-.13)$ was higher than ROM $(\beta=-.06)$ (Wibault et al., 2014). In a cross-sectional study which used principal components analysis (PCA), the first component which explained $56 \%$ of the data's variance had a high weighting for neck muscle endurance (Halvorsen et al., 2015). A limitation of using a PCA is that it does not model the relationship between the extracted components on the outcome of disability. Peolsson and Peolsson (2008) reported in a prospective analysis that cervical ROM and hand grip strength were important predictors of long-term (range: 56-94 months) disability. However, a limitation of the study was that psychological factors were not included in the model (Peolsson \& Peolsson, 2008).

The findings of the present study provide unique insights which could optimize post-surgical management of patients with CR. Although factors such as depression, cervical ROM and neck muscle endurance have been suggested to be important variables (Engquist et al., 2015; Li et al., 2015; Peolsson et al., 2013, 2006; Persson \& Lilja, 2001; Skeppholm et al., 2017; Wibault et al., 2018, 2017), the present study suggests that therapeutic efforts could be aimed at improving self-efficacy levels within the first 6 months post-surgery to improve pain-related disability in individuals with CR. When designing future intervention studies, our findings would predict that two interventions with similar effects on self-efficacy would have similar effects on the change in pain-related disability in post-surgical individuals with CR. In addition, interventions designed to enhance self-efficacy may be better tested on a cohort with low baseline self-efficacy levels_otherwise, a ceiling effect of improvement in self-efficacy would be reached. A hypothetical scenario could be that when baseline self-efficacy level is normal, SES_d6 tends towards a fixed value of zero, and arrows leading out of SES_d6 are removed. This means that the primary driver of recovery in disability would be the reduction of neck pain.

Our findings should not be misconstrued to suggest that only cognitive-based approaches, and not physical-based approaches should be used in the rehabilitation of post-surgical individuals with CR. Physical-based approaches, when framed as a means of reconceptualizing the painful disorder while normalizing painful postures and movements, have been shown to reduce pain (Vibe Fersum, O'Sullivan, Skouen, Smith, \& Kvale, 2013; Vibe Fersum, Smith, Kvale, Skouen, \& O'Sullivan, 2019) and disability (Vibe Fersum et al., 2013, 2019), and improve self-efficacy (O'Sullivan, Dankaerts, O'Sullivan, \& O'Sullivan, 2015). 
Given that the predictive correlation of the model was not high across all variables, it suggests some limitations of the model. A limitation may be that we may not have included all candidate variables into the BN model. The model's predictive performance and the learnt dependency relationships may also be influenced by the sample size of the cohort (see range of sample sizes in [Holla et al., 2014]), and the stage of recovery of a disorder. The variables included in the current study were based on prior knowledge about their mediating and prognostic value. Realistically, the number of variables included into a BN model must depend not only on prior knowledge but should also consider the logistical feasibility of measuring these measures in a busy clinical environment. Hence, we view the relationships learnt in this study within a "hypothesis-generation" framework, where plausible mediators identified could be targets of intervention in future randomized controlled studies. Future mediation studies would benefit from the present study's methods, given the capacity to build and compare competing models, to evaluate which model best fits the data.

\section{CONCLUSIONS}

Presently, there is little high-quality evidence from RCTs to guide the optimal post-operative management in individuals with CR. Improvement in self-efficacy was the primary driver influencing the change in neck-pain disability post-operation. Changes in self-efficacy levels at 6-month post-surgery had both a direct influence and an indirect influence via the mediation of changes in neck and arm pain at 6 and 12 months, on the change in disability in individuals with CR. The present study provides potentially modifiable mediators that could be the target of future intervention trials. BN models could increase the precision of treatment and outcome assessment of individuals with CR.

\section{CONFLICTS OF INTEREST}

The authors have no conflicts of interest to declare.

\section{Funding Information}

\begin{tabular}{l} 
Swedish Research Council \\
FunderDOI : $10.13039 / 501100004359$ \\
\hline Swedish Society of Medicine \\
FunderDOI : $10.13039 / 501100007687$ \\
\hline $\begin{array}{l}\text { Medical Research Council of Southeast Sweden } \\
\text { FunderDOI : } 10.13039 / 100010805\end{array}$ \\
\hline Region Östergötland \\
\hline Lions \\
\hline Futurum \\
\hline
\end{tabular}

\section{Supplementary Material}




\section{REFERENCES}

Altmaier, E. M., Russell, D. W., Kao, C. F., Lehmann, T. R., \& Weinstein, J. N. (1993). Role of self-efficacy in rehabilitation outcome among chronic low back pain patients. Journal of Counseling Psychology, 40, 335-339. https://doi.org/10.1037/0022-0167.40.3.335

Andersen, T. E., Karstoft, K.-I., Brink, O., \& Elklit, A. (2016). Pain-catastrophizing and fear-avoidance beliefs as mediators between post-traumatic stress symptoms and pain following whiplash injury - A prospective cohort study. European Journal of Pain, 20, 1241-1252. https://doi.org/10.1002/ejp.848

Andresen, A. K., Paulsen, R. T., Busch, F., Isenberg-Jorgensen, A., Carreon, L. Y., \& Andersen, M. O. (2018). Patient-reported outcomes and patient-reported satisfaction after surgical treatment for cervical radiculopathy. Global Spine Journal, 8, 703-708. https://doi.org/10.1177/2192568218765398

Arnstein, P., Caudill, M., Mandle, C. L., Norris, A., \& Beasley, R. (1999). Self efficacy as a mediator of the relationship between pain intensity, disability and depression in chronic pain patients. Pain, 80,483-491. https://doi.org/10.1016/S0304-3959(98)002206

Bono, C. M., Ghiselli, G., Gilbert, T. J., Kreiner, D. S., Reitman, C., Summers, J. T., ... Lamer, T. (2011). An evidence-based clinical guideline for the diagnosis and treatment of cervical radiculopathy from degenerative disorders. The Spine Journal, 11, 64-72. https://doi.org/10.1016/j.spinee.2010.10.023

Bunketorp, L., Carlsson, J., Kowalski, J., \& Stener-Victorin, E. (2005). Evaluating the reliability of multi-item scales: A non-parametric approach to the ordered categorical structure of data collected with the Swedish version of the Tampa Scale for Kinesiophobia and the Self-Efficacy Scale. Journal of Rehabilitation Medicine, 37, 330334. https://doi.org/10.1080/16501970510036411

Cheng, S.-T., Leung, C. M. C., Chan, K. L., Chen, P. P., Chow, Y. F., Chung, J. W. Y., ... Tam, C. W. C. (2018). The relationship of self-efficacy to catastrophizing and depressive symptoms in community-dwelling older adults with chronic pain: A moderated mediation model. PLOS ONE, 13, e0203964.

https://doi.org/10.1371/journal.pone.0203964

Craig, A., Tran, Y., Siddall, P., Wijesuriya, N., Lovas, J., Bartrop, R., \& Middleton, J. (2013). Developing a model of associations between chronic pain, depressive mood, chronic fatigue, and self-efficacy in people with spinal cord injury. The Journal of Pain, 14, 911920. https://doi.org/10.1016/j.jpain.2013.03.002

Engquist, M., Löfgren, H., Öberg, B., Holtz, A., Peolsson, A., Söderlund, A., ... Lind, B. (2015). Factors affecting the outcome of surgical versus nonsurgical treatment of cervical radiculopathy: A randomized, controlled study. Spine, 40, 1553-1563. https://doi.org/10.1097/BRS.0000000000001064

Farmer, N. (2014). An update and further testing of a knowledge-based diagnostic clinical decision support system for musculoskeletal disorders of the shoulder for use 
in a primary care setting. Journal of Evaluation in Clinical Practice, 20, 589-595. https://doi.org/10.1111/jep.12153

Fordham, B., Ji, C., Hansen, Z., Lall, R., \& Lamb, S. E. (2017). Explaining how cognitive behavioral approaches work for low back pain: Mediation analysis of the back skills training trial. Spine, 42, E1031-E1039. https://doi.org/10.1097/BRS.0000000000002066

Friedman, N. (1997). Learning belief networks in the presence of missing values and hidden variables. Proceedings of the Fourteenth International Conference on Machine Learning. Morgan Kaufmann Publishers Inc.; 125-133.

Gross, A., Kay, T. M., Paquin, J.-P., Blanchette, S., Lalonde, P., Christie, T., ... Santaguida, P. L. (2015). Exercises for mechanical neck disorders. Cochrane Database of Systematic Reviews, 1, Cd004250. https://doi.org/10.1002/14651858.CD004250.pub5

Hall, A. M., Kamper, S. J., Emsley, R., \& Maher, C. G. (2016). Does pain-catastrophising mediate the effect of tai chi on treatment outcomes for people with low back pain? Complementary Therapies in Medicine, 25, 61-66.

https://doi.org/10.1016/j.ctim.2015.12.013

Halvorsen, M., Kierkegaard, M., Harms-Ringdahl, K., Peolsson, A., \& Dedering, Å. (2015). Dimensions underlying measures of disability, personal factors, and health status in cervical radiculopathy: A cross-sectional study. Medicine (Baltimore), 94, e999-e999. https://doi.org/10.1097/MD.0000000000000999

Hermansen, A. M., Cleland, J. A., Kammerlind, A. S., \& Peolsson, A. L. (2014). Evaluation of physical function in individuals 11 to 14 years after anterior cervical decompression and fusion surgery-A comparison between patients and healthy reference samples and between 2 surgical techniques. Journal of Manipulative \& Physiological Therapeutics, 37, 87-96. https://doi.org/10.1016/j.jmpt.2013.11.002

Hermansen, A., Hedlund, R., Vavruch, L., \& Peolsson, A. (2011). A comparison between the carbon fiber cage and the cloward procedure in cervical spine surgery: $A$ ten- to thirteen-year follow-up of a prospective randomized study. Spine, 36, 919-925. https://doi.org/10.1097/BRS.0b013e3181e8e4a3

Hinkle, D., Wiersma, W., \& Jurs, S. (2003). Applied statistics for the behavioral sciences. $\mathrm{AQ}_{4}$ 5. Boston: Houghton Mifflin.

Holla, J. F. M., Sanchez-Ramirez, D. C., van der Leeden, M., Ket, J. C. F., Roorda, L. D., Lems, W. F., ... Dekker, J. (2014). The avoidance model in knee and hip osteoarthritis: A systematic review of the evidence. Journal of Behavioral Medicine, 37, 1226-1241. https://doi.org/10.1007/s10865-014-9571-8

Jensen, I. B., \& Linton, S. J. (1993). Coping Strategies Questionnaire (CSQ): Reliability of the Swedish version of the CSQ. Scandinavian Journal of Behaviour Therapy, 22, 139145. https://doi.org/10.1080/16506079309455940

Lee, H., Hubscher, M., Moseley, G. L., Kamper, S. J., Traeger, A. C., Mansell, G., \& McAuley, J. H. (2015). How does pain lead to disability? A systematic review and meta- 
analysis of mediation studies in people with back and neck pain. Pain, 156, 988-997. https://doi.org/10.1097/j.pain.0000000000000146

Li, S., Qi, M., Yuan, W., \& Chen, H. (2015). The impact of the depression and anxiety on prognosis of cervical total disc replacement. Spine, 40, E266-E271.

https://doi.org/10.1097/BRS.0000000000000743

Main, C. J. (1983). The modified somatic perception questionnaire (MSPQ). Journal of Psychosomatic Research, 27, 503-514. https://doi.org/10.1016/0022-3999(83)90040-5

Mansell, G., Hill, J. C., Main, C., Vowles, K. E., \& van der Windt, D. (2016). Exploring what factors mediate treatment effect: Example of the STarT Back Study High-Risk Intervention. The Journal of Pain, 17, 1237-1245.

https://doi.org/10.1016/j.jpain.2016.08.005

Mansell, G., Kamper, S. J., \& Kent, P. (2013). Why and how back pain interventions work: What can we do to find out? Best Practice \& Research Clinical Rheumatology, 27, 685697. https://doi.org/10.1016/j.berh.2013.10.001

Marshall, P. W. M., Schabrun, S., \& Knox, M. F. (2017). Physical activity and the mediating effect of fear, depression, anxiety, and catastrophizing on pain related disability in people with chronic low back pain. PLOS ONE, 12, e0180788. https://doi.org/10.1371/journal.pone.0180788

Monticone, M., Cedraschi, C., Ambrosini, E., Rocca, B., Fiorentini, R., Restelli, M., ... Moja, L. (2015). Cognitive-behavioural treatment for subacute and chronic neck pain. Cochrane Database of Systematic Reviews, Cd010664. https://doi.org/10.1002/14651858.CD010664.pub2

Murray, C. J., Atkinson, C., Bhalla, K., Birbeck, G., Burstein, R., Chou, D., ... Murray (2013). The state of US health, 1990-2010: Burden of diseases, injuries, and risk factors. Journal of the American Medical Association, 310, 591-608.

AQ5 https://doi.org/10.1001/jama.2013.13805

Nagarajan, R., Scutari, M., \& Lèbre, S. (2013). Bayesian networks in $R$ with applications in systems biology. New York: Springer Verlag.

Nieto, R., Miro, J., \& Huguet, A. (2009). The fear-avoidance model in whiplash injuries. European Journal of Pain, 13, 518-523. https://doi.org/10.1016/j.ejpain.2008.06.005

O'Sullivan, K., Dankaerts, W., O'Sullivan, L., \& O'Sullivan, P. B. (2015). Cognitive functional therapy for disabling nonspecific chronic low back pain: Multiple case-cohort study. Physical Therapy, 95, 1478-1488. https://doi.org/10.2522/ptj.20140406

Parker, S. L., Godil, S. S., Shau, D. N., Mendenhall, S. K., \& McGirt, M. J. (2013). Assessment of the minimum clinically important difference in pain, disability, and quality of life after anterior cervical discectomy and fusion: Clinical article. Journal of Neurosurgery Spine, 18, 154-160. https://doi.org/10.3171/2012.10.SPINE12312

Passias, P. G., Hasan, S., Radcliff, K., Isaacs, R., Bianco, K., Jalai, C. M., ... Gerling, M. C. (2018). Arm pain versus neck pain: A novel ratio as a predictor of post-operative clinical 
outcomes in cervical radiculopathy patients. International Journal of Spine Surgery, 12, 629-637. https://doi.org/10.14444/5078

Pedler, J. A., Kamper, J. S., Maujean, J. A., \& Sterling, J. M. (2018). Investigating the fear avoidance model in people with whiplash: The association between fear of movement and in vivo activity. The Clinical Journal of Pain, 34, 130-137.

https://doi.org/10.1097/AJP.0000000000000524

Peolsson, A., Öberg, B., Wibault, J., Dedering, A., Zsigmond, P., Bernfort, L., ... Löfgren, H. (2014). Outcome of physiotherapy after surgery for cervical disc disease: $A$ prospective randomised multi-centre trial. BMC Musculoskeletal Disorders, 15, 34. https://doi.org/10.1186/1471-2474-15-34

Peolsson, A., \& Peolsson, M. (2008). Predictive factors for long-term outcome of anterior cervical decompression and fusion: A multivariate data analysis. European Spine Journal, 17, 406-414. https://doi.org/10.1007/s00586-007-0560-2

Peolsson, A., Söderlund, A., Engquist, M., Lind, B., Löfgren, H., Vavruch, L., ... Öberg, B. (2013). Physical function outcome in cervical radiculopathy patients after physiotherapy alone compared with anterior surgery followed by physiotherapy: A prospective randomized study with a 2-year follow-up. Spine, 38, 300-307. https://doi.org/10.1097/BRS.0b013e31826d2cbb

Peolsson, A., Vavruch, L., \& Öberg, B. (2002). Disability after anterior decompression and fusion for cervical disc disease. Advances in Physiotherapy, 4, 111-124. https://doi.org/10.1080/140381902320387531

Peolsson, A., Vavruch, L., \& Öberg, B. (2006). Predictive factors for arm pain, neck pain, neck specific disability and health after anterior cervical decompression and fusion. Acta Neurochirurgica, 148, 167-173. https://doi.org/10.1007/s00701-005-0660-x

Persson, L. C. G., \& Lilja, A. (2001). Pain, coping, emotional state and physical function in patients with chronic radicular neck pain. A comparison between patients treated with surgery, physiotherapy or neck collar - A blinded, prospective randomized study. Disability and Rehabilitation, 23, 325-335. https://doi.org/10.1080/09638280010005567

Radhakrishnan, K., Litchy, W. J., O'Fallon, W. M., \& Kurland, L. T. (1994) Epidemiology of cervical radiculopathy. A population-based study from Rochester, Minnesota, 1976 through 1990. Brain, 117(Pt 2), 325-335.

Saunders, S. W., Schache, A., Rath, D., \& Hodges, P. W. (2005). Changes in three dimensional lumbo-pelvic kinematics and trunk muscle activity with speed and mode of locomotion. Clinical Biomechanics, 20, 784-793. https://doi.org/10.1016/j.clinbiomech.2005.04.004

Scutari, M. (2010). Learning Bayesian networks with the bnlearn R Package. Journal of Statistical Software, 35, 22.

Scutari, M., Auconi, P., Caldarelli, G., \& Franchi, L. (2017). Bayesian networks analysis of malocclusion data. Scientific Reports, 7, 15236. https://doi.org/10.1038/s41598-01715293-w 
Scutari, M., \& Nagarajan, R. (2013). Identifying significant edges in graphical models of molecular networks. Artificial Intelligence in Medicine, 57, 207-217.

https://doi.org/10.1016/j.artmed.2012.12.006

Sesen, M. B., Nicholson, A. E., Banares-Alcantara, R., Kadir, T., \& Brady, M. (2013). Bayesian networks for clinical decision support in lung cancer care. PLOS ONE, 8, e82349. https://doi.org/10.1371/journal.pone.0082349

Skeppholm, M., Fransson, R., Hammar, M., \& Olerud, C. (2017). The association between preoperative mental distress and patient-reported outcome measures in patients treated surgically for cervical radiculopathy. The Spine Journal, 17, 790-798. https://doi.org/10.1016/j.spinee.2016.02.037

Skidmore, J. R., Koenig, A. L., Dyson, S. J., Kupper, A. E., Garner, M. J., \& Keller, C. J. (2015). Pain self-efficacy mediates the relationship between depressive symptoms and pain severity. The Clinical Journal of Pain, 31, 137-144.

https://doi.org/10.1097/AJP.0000000000000094

Sleijser-Koehorst, M. L. S., Coppieters, M. W., Heymans, M. W., Rooker, S., Verhagen, A. P., \& Scholten-Peeters, G. G. M. (2018). Clinical course and prognostic models for the conservative management of cervical radiculopathy: A prospective cohort study. European Spine Journal, 27, 2710-2719. https://doi.org/10.1007/s00586-018-5777-8

Söderlund, A., \& Åsenlöf, P. (2010). The mediating role of self-efficacy expectations and fear of movement and (re)injury beliefs in two samples of acute pain. Disability and Rehabilitation, 32, 2118-2126. https://doi.org/10.3109/09638288.2010.483036

Soderlund, A. P., Sandborgh, M. P., \& Johansson, A. C. P. (2017). Is self-efficacy and catastrophizing in pain-related disability mediated by control over pain and ability to decrease pain in whiplash-associated disorders? Physiotherapy Theory and Practice, 33, 376-385. https://doi.org/10.1080/09593985.2017.1307890

Spinhoven, P., ter Kuile, M., Kole-Snijders, A. M. J., Hutten Mansfeld, M., den Ouden, D.J., \& Vlaeyen, J. W. S. (2004). Catastrophizing and internal pain control as mediators of outcome in the multidisciplinary treatment of chronic low back pain. European Journal of Pain, 8, 211-219. https://doi.org/10.1016/j.ejpain.2003.08.003

Steinhaus, M. E., Iyer, S., Lovecchio, F., Stein, D., Ross, T., Yang, J., ... Kim, H. J. (2019). Which NDI domains best predict change in physical function in patients undergoing cervical spine surgery? The Spine Journal, 19, 1698-1705.

https://doi.org/10.1016/j.spinee.2019.06.006

Takenaka, S., \& Aono, H. (2017). Prediction of postoperative clinical recovery of drop foot attributable to lumbar degenerative diseases, via a Bayesian network. Clinical Orthopaedics and Related Research, 475, 872-880. https://doi.org/10.1007/s11999016-5180-x

Vibe Fersum, K., O'Sullivan, P., Skouen, J. S., Smith, A., \& Kvale, A. (2013). Efficacy of classification-based cognitive functional therapy in patients with non-specific chronic low back pain: A randomized controlled trial. European Journal of Pain, 17, 916-928. https://doi.org/10.1002/j.1532-2149.2012.00252.x 
Vibe Fersum, K., Smith, A., Kvale, A., Skouen, J. S., \& O'Sullivan, P. (2019). Cognitive functional therapy in patients with non-specific chronic low back pain-A randomized controlled trial 3-year follow-up. European Journal of Pain, 23(8), 1416-1424. https://doi.org/10.1002/ejp.1399

Whittle, R., Mansell, G., Jellema, P., \& van der Windt, D. (2017). Applying causal mediation methods to clinical trial data: What can we learn about why our interventions (don't) work? European Journal of Pain, 21, 614-622.

https://doi.org/10.1002/ejp.964

Wibault, J., Oberg, B., Dedering, A., Lofgren, H., Zsigmond, P., \& Peolsson, A. (2018). Structured postoperative physiotherapy in patients with cervical radiculopathy: 6month outcomes of a randomized clinical trial. Journal of Neurosurgery Spine, 28, 1-9. https://doi.org/10.3171/2017.5.SPINE16736

Wibault, J., Öberg, B., Dedering, Å., Löfgren, H., Zsigmond, P., Persson, L., ... Peolsson, A. (2017). Neck-related physical function, self-efficacy, and coping strategies in patients with cervical radiculopathy: A randomized clinical trial of postoperative physiotherapy. Journal of Manipulative \& Physiological Therapeutics, 40, 330-339. https://doi.org/10.1016/j.jmpt.2017.02.012

Wibault, J., Öberg, B., Dedering, Å., Löfgren, H., Zsigmond, P., Persson, L., \& Peolsson, A. (2014). Individual factors associated with neck disability in patients with cervical radiculopathy scheduled for surgery: A study on physical impairments, psychosocial factors, and life style habits. European Spine Journal, 23, 599-605.

https://doi.org/10.1007/s00586-013-3066-0

Wicksell, R. K., Olsson, G. L., \& Hayes, S. C. (2010). Psychological flexibility as a mediator of improvement in Acceptance and Commitment Therapy for patients with chronic pain following whiplash. European Journal of Pain, 14, 1059.e1-1059.e11.

https://doi.org/10.1016/j.ejpain.2010.05.001

Ylinen, J. J., Savolainen, S., Airaksinen, O., Kautiainen, H., Salo, P., \& Häkkinen, A. (2003). Decreased strength and mobility in patients after anterior cervical diskectomy compared with healthy subjects 11 No commercial party having a direct financial interest in the results of the research supporting this article has or will confer a benefit on the authors or on any organization with which the authors are associated. Archives of Physical Medicine and Rehabilitation, 84, 1043-1047. https://doi.org/10.1016/S00039993(03)00039-X

Zhang, Y., Zhang, T., Zhang, C., Tang, F., Zhong, N., Li, H., ... Xue, F. (2015). Identification of reciprocal causality between non-alcoholic fatty liver disease and metabolic syndrome by a simplified Bayesian network in a Chinese population. British Medical Journal Open, 5, e008204. https://doi.org/10.1136/bmjopen-2015-008204

Zung, W. W. (1965). A self-rating depression scale. Archives of General Psychiatry, 12, 63-70. https://doi.org/10.1001/archpsyc.1965.01720310065008 